\title{
MODELING OF A TURBO CHARGED SI ENGINE
}

\section{Lars Eriksson, Lars Nielsen, Jan Brugård, Johan Bergström, Fredrik Pettersson, and Per Andersson}

\author{
Vehicular Systems, Linköping University \\ SE-581 83 Linköping, SWEDEN \\ Tel: +46-13-284409, Fax:+46-13-282035 \\ E-mail: larer@isy.liu.se, lars@isy.liu.se
}

\begin{abstract}
Turbo charged SI engines are a major possibility in the current trend of down-sized engines with preserved drivability performance. Considering control and supervision it is favorable to have a mean value model to be used e.g. in observer design. Such models of turbo engines are similar to those of naturally aspirated engines, but there are some special characteristics, e.g. the interconnected gas flows, the intercooler, the difference in relative sizes between the gas volumes (compared to naturally aspirated engines), the turbo, and the waste gate. Here, a model is developed with a strategy to find a model for each engine component (air filter, compressor, after cooler (or intercooler), throttle, engine, turbine, waste gate, and a lumped model for the catalyst and exhaust) as they behave in an engine setting. When investigating agreement with measured data and sensitivity of possible model structures, a number of interesting issues are raised. The experiments and the model validation have been performed on a Saab 2.31 production engine.
\end{abstract}

Keywords: Automotive control, compressor/turbine modeling, intercooler modeling.

\section{INTRODUCTION}

Mean value models of SI engines have been successfully utilized in several aspects of engine control, engine management, and engine supervision. These mean value models are usually based on non-linear ordinary differential equations, as opposed to wave models using partial differential equations that are used for more detailed (and computationally expensive) modeling. Mean value models are favorable for design of control and supervision systems, where they form an excellent basis for e.g. observer design.

Looking at models of turbo engines, the situation is different for CI and SI engines. Models for turbo charged CI engines have been treated for a long time due to the importance of the turbocharger on $\mathrm{CI}$ engine performance. Nowadays, almost all modern diesel engine cars are equipped with a turbocharger since it at the same time improves both driveability and emissions from the engine. For SI engines the scene has been a little different. The emissions are effectively handled by the three-way catalyst, and the turbo charger has accordingly been seen as a luxury device for power boost. However, this viewpoint is now changing since the drive towards lowering $\mathrm{CO}_{2}$ is enforcing down sizing concepts for SI engines. With a down sizing concept the engine size is reduced in order to reduce the pumping losses at part load. The result could unfortunately be a lower power output, so to overcome this a supercharger is usually fitted which increases the power of the engine. See e.g. (Guzzella et al., 2000) for a more thorough motivation and discussion on down sizing.

This paper is organized so that the next section gives an overview of the engine, its components, and the modeling strategy. The following sections then treat component by component. We only give a summary of the models that we have considered/developed, but the full derivations can be found in (Bergström and Brugård, 1999; Pettersson, 2000). 


\section{ENGINE MODELING}

In Fig. 1 a sketch of a turbo charged SI engine is displayed. The figure shows the components that have to be modeled. They are: air filter, compressor, after cooler (or intercooler), throttle, engine, turbine, waste gate, and a lumped model for the catalyst and exhaust.

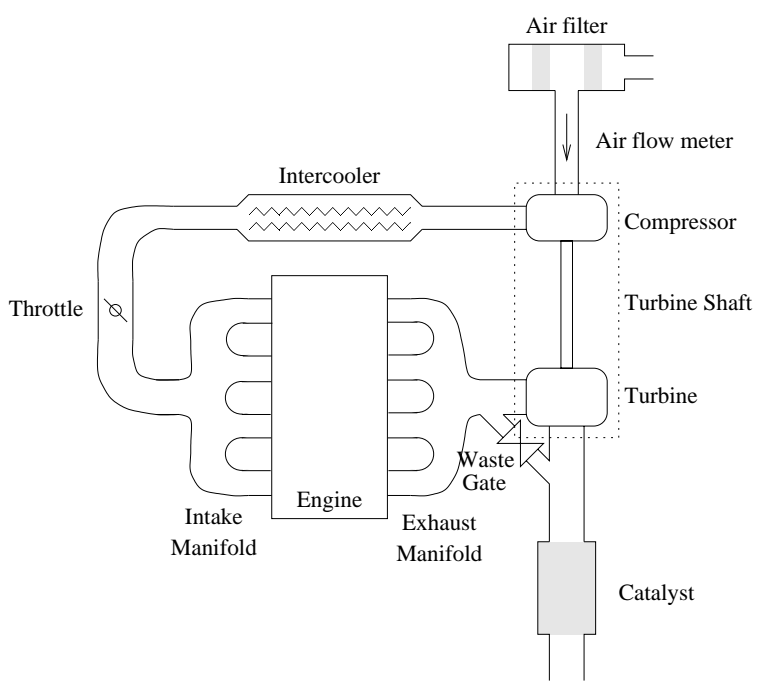

Fig. 1. Sketch of a turbo charged SI-engine. The figure illustrates how the engine is divided into subsystems to enable physical modeling.

\subsection{Modeling strategy}

A component based modeling strategy is used. For each component, there already exist models of different complexity in the literature. The question we have asked ourselves is how well these different possibilities fit in the framework of mean value modeling for control and supervision. A first issue is of course how well measured data can be represented and what the characteristics of the modeling errors are. Included here is a question of complexity. For example, as will be studied below, is it enough with a simple model for a flow restriction or should d'Arcys law be included. Another issue is about model structures and their sensitivity e.g. to changes in operation conditions of the engine. One example here is that even though compressor maps can be mathematically inverted to give mass flow as a function of pressure ratio, it is practically useless since the speed lines becomes almost vertical. Here, one may instead seek regression models, hopefully with cleverly chosen regressors, that form tractable local inverses. Of course, it has then to be experimentally verified that the compressor, in its engine setting, operates within the validity region for the regressor model.

The result of such a modeling investigation is a set of model structures that can be fitted to an actual engine (or engine family), and then fulfills the requirements for e.g. observer design.
2.1.1. Mean value models We assume that the reader is familiar with mean value engine models. The basic principle used here is that each engine component shown in Fig. 1 is modeled as a flow restriction (or pump) that affects the thermodynamic state of the fluid (gaseous). The standard calculation causality of almost all restrictions is that they take pressure change as input and gives the flow as a result. The volumes between the restrictions are modeled as reservoirs where normally the pressure of each reservoir represent a state in the model. As a side note: We have also tested modern modeling tools based on object orientation and equations. There the causality is handled automatically, but it is still important to consider model structures, for example to avoid sensitivity problems as mentioned above.

2.1.2. Abbreviated Nomenclature It is natural to accompany the component based modeling with a system to name the variables. The main variables in the table to the left are specified by a subscript from the table to the right referring to its own component or the component before. As an example, $T_{c}$ refers to the temperature after the compressor.

\begin{tabular}{|c|l|}
\hline \multicolumn{2}{|c|}{ Variables } \\
\hline$P$ & Power \\
$p$ & Pressure \\
$T$ & Temperature \\
$T q$ & Torque \\
\hline
\end{tabular}

\begin{tabular}{|c|l|}
\hline \multicolumn{2}{|c|}{ Subscripts } \\
\hline a & Ambient \\
af & Air filter \\
c & Compressor \\
em & Exhaust manifold \\
es & Exhaust system \\
ic & Intercooler \\
im & Intake manifold \\
t & Turbine \\
th & Throttle \\
\hline
\end{tabular}

2.1.3. Measurements and validation The experiments and the model validation have been performed on a Saab 2.31 production engine. The figures showing the model validation are slightly scaled so that the performance of the engine will not be revealed. This is done as a courtesy to our collaboration partners. However this doesn't restrict our observations since we are focusing on the model structures and their properties.

\subsubsection{Comments on pressure measurement When} applying mean value models the focus is on deriving models that describe the system on an actuator to sensor or sensor to sensor perspective. Therefore pressure models are developed mainly for static pressure since the pressure sensors are usually mounted such that they measure the static pressure. This choice can be further supported since the dynamic pressure in the inlet system is usually lower than $0.4 \%$ of total pressure.

One important step in the modeling of the restrictions is the accuracy or calibration of the pressure sensors. Two approaches were used: 1) With the engine shut 
off a measurement is made and an offset among the sensors, at atmospheric conditions, can be detected. 2) An extra sensor model in which an offset is added to one or more of the sensors. Both these methods were applied and produced the same sensor offset. The difference between them was less than $100 \mathrm{~Pa}$. A third method is to mount all sensors via pipes to the same position in the intake manifold and then make measurements with a running engine over the whole operating range of the intake manifold pressure. Using this procedure all sensors can be compared and each individual sensor can be calibrated to yield the same values as the other for the same conditions.

\section{FLOW RESTRICTIONS}

In many engine components the fluid flow can be regarded as incompressible and in-viscid. Under such conditions the pressure head losses caused by bends, valves, and sudden changes in area approximately follow the same relation

$$
\Delta p_{\mathrm{f}}=\xi(R e) \frac{\rho V_{1}^{2}}{2}=\xi(R e) \frac{R T_{1} \dot{m}^{2}}{p_{1}}
$$

where the $\xi$ only has a weak dependence on the Reynolds number (Massey, 1998). In most cases $\xi(R e)$ can be regarded as constant (here denoted $\mathcal{H}$ ), and the equation can be used as a general model for incompressible flow through a restriction. Models for the air filter, intercooler, and exhaust system are all based on this relation. However, if the model (1) with a pure second order term is not sufficient to describe the head loss, then a linear term corresponding to d'Arcys law for head losses accompanying flow through thin pipes or porous media can be added.

\subsection{Air Filter - Pressure Head Loss}

The air filter is important to model since it produces a pressure drop that can be as high as $5 \%$, which propagates trough the intake system and influences the whole engine. A sketch of the air filter is shown in Fig. 2. It consists of three sudden changes in area, the filter itself, and the pipes in and out of it. As described above, Eq. (1) can be used to model pressure loss for several kinds of flow restrictions, which gives the following pressure loss over the air filter:

$$
\Delta p_{\mathrm{af}}=\mathcal{H}_{\mathrm{af}} \frac{T_{\mathrm{a}} \dot{m}_{\mathrm{air}}^{2}}{p_{\mathrm{a}}}
$$

3.1.1. Validation A least square fit to measured data, gives the constant $\mathcal{H}_{\mathrm{af}}$ for Eq. (2). In Fig. 3 the model is represented by a solid line, and the validation data is superimposed. As can be clearly seen in the figure this model describes the pressure loss very accurately; the average error is $0.05 \mathrm{kPa}$ and the maximum error is less then $0.16 \mathrm{kPa}$. It should

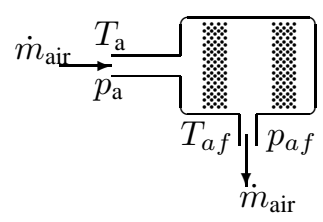

Fig. 2. Sketch of the air filter showing the changes in flow area.

be noted that the external environment of the engine could not be manipulated i.e. changes were not made in temperature, pressure, or humidity at the test bench. Consequently, there has been no possibility to validate the model for different ambient conditions.

\subsection{Exhaust-Pressure Head Loss}

The exhaust system consists of changes in flow area and flow direction and the head losses can therefore also be modeled in the same way as the air filter, i.e. using Eq. (1), with a constant $\xi$. The model is validated in Fig. 3 and it shows good agreement with validation data, with an average error of $0.5 k P a$ and maximum error less then $4.2 \mathrm{kPa}$.
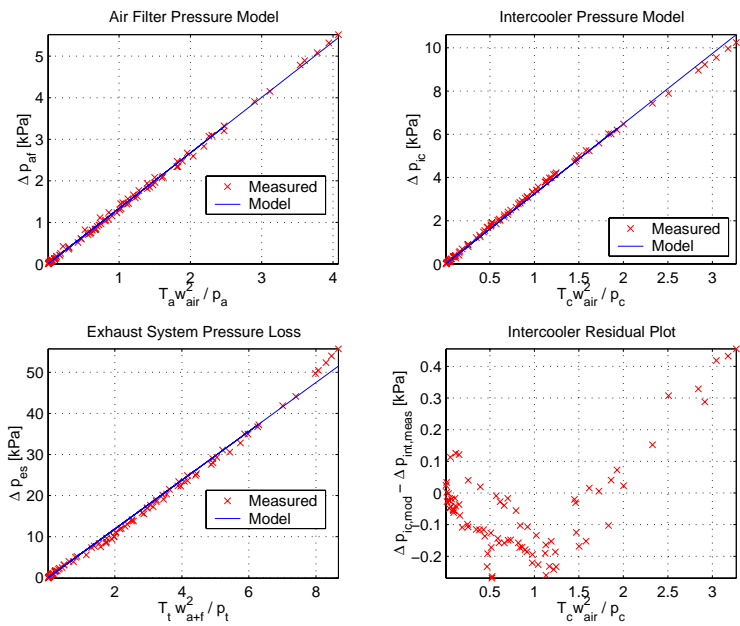

Fig. 3. Validation of pressure losses over the restrictions. Top left - air filter. Bottom left - exhaust system. Top right - intercooler. Bottom right intercooler residuals. The corrected air mass flow is given by $\frac{T \dot{m}^{2}}{p}$.

\section{INTERCOOLER}

A heat exchanger, called intercooler, is used after the compressor to cool the charge air back to near ambient temperature. It increases air density which is advantageous, but in SI engines it is mainly used for knock reduction. In engines the most common heat exchanger is the cross flow intercooler with both fluids unmixed which is shown in Fig. 4. To achieve efficient cooling of the charge air, the tubes in the intercooler need to be rather thin so that the air is exposed as much as possible to the cooling medium. The result 
is that some of the gain in intake air density is lost. Therefore, beside the obvious ned to model the outlet air temperature, also a model for the pressure head loss is needed.

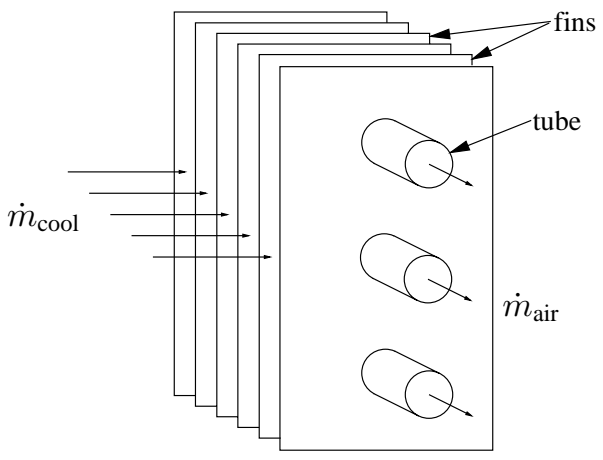

Fig. 4. Cross flow, both fluids unmixed intercooler.

\subsection{Intercooler - Pressure Head Loss}

The air path through the intercooler passes a number of area changes and through thin and long tubes. This motivates that the head losses can be modeled in the same way as for the air filter, (1). However, since the flow also passes through some thin pipes also a linear term, according to d'Arcys law, may improve the accuracy

$$
\Delta p_{\mathrm{ic}}=\mathcal{H}_{\mathrm{ic}, 1} \frac{T_{\mathrm{c}} \dot{m}_{\mathrm{air}}}{p_{\mathrm{c}}}+\mathcal{H}_{\mathrm{ic}, 2} \frac{T_{\mathrm{c}} \dot{m}_{\mathrm{air}}^{2}}{p_{\mathrm{c}}}
$$

4.1.1. Validation A least squares fit to measured data, gives the constants $\mathcal{H}_{\text {ic }}$ in Eqs (1) and (3). In Fig. 3 the model with a quadratic term is validated and it is seen that it has a systematic error with respect to the air flow. This motivates the addition of a linear term which improves the fit. With a linear term (not shown) the maximum error is reduced from $400 \mathrm{~Pa}$ to $200 \mathrm{~Pa}$, and the systematic error is reduced. As can be clearly seen in the figure, even Model (1) describes the pressure loss very accurately with an average error around $100 \mathrm{~Pa}$, which usually is sufficient.

\subsection{Temperature Models}

For all practical purposes of intercoolers the flow rate of the cooling fluid $\dot{m}_{\text {cool }}$ is greater than the air mass flow, $\dot{m}_{\text {air }}$, through it. This suggests, (Holman, 1992), that the following equation can be used to measure the efficiency of the intercooler

$$
\varepsilon=\frac{T_{\mathrm{c}}-T_{\mathrm{ic}}}{T_{\mathrm{c}}-T_{\text {cool }}}
$$

Solving this equation for $T_{\text {ic }}$ yields the desired expression for the intercooler outlet temperature, expressed in terms of the temperatures and the intercooler efficiency

$$
T_{\mathrm{ic}}=T_{\mathrm{c}}-\varepsilon\left(T_{\mathrm{c}}-T_{\mathrm{cool}}\right)
$$

In order to predict the intercooler outlet temperature, a model for the intercooler efficiency, $\varepsilon$, is needed. Two models of different complexity are considered; first the standard NTU-model (see e.g. (Holman, 1992) for a derivation), and then a regression model.

4.2.1. NTU model A derivation and discussion on how to apply the NTU-model to an automotive intercooler is given in (Bergström and Brugård, 1999). The model equations are arranged as follows

$$
\begin{aligned}
\varepsilon & =1-e^{\frac{e^{-N^{0.78} C_{-1}}}{N^{-0.22 C}}} \\
N & =\frac{U A}{c_{\text {p,air }} \dot{m}_{\text {air }}}=\frac{K}{c_{\text {p,air }}} \dot{m}_{\text {air }}^{-0.2} \mu_{i}^{-0.5} \\
\mu_{i} & =2.3937 \cdot 10^{-7}\left(\frac{T_{\mathrm{c}}+T_{\text {cool }}}{2}\right)^{0.7617} \\
C & =\frac{\dot{m}_{\text {air }}}{\dot{m}_{\text {cool }}}
\end{aligned}
$$

The unknown constant $K$ is determined from a least squares fit to measured data, and as can be seen, the model is expressed in variables that are easily determined. The grouping $\frac{U A}{c_{\mathrm{p}, \text { air }}}$ is called the number of heat transfer units (NTU), and therefore the above model is referred to as the NTU-model.

4.2.2. Regression efficiency model The physics of heat transfer is built into the NTU-model but its structure is complex, which motivates the search for other models. Another approach to model $\varepsilon$ is to use the heat transfer considerations made when developing the NTU-model as an indicator of what parameters effect the efficiency and use them as regressors in a linear regression model. Choosing the mean tube temperature and air mass flow together with the ratio of the air mass flow and the cooling air mass flow, the following model for $\varepsilon$ may be stated

$$
\varepsilon=a_{0}+a_{1}\left(\frac{T_{\mathrm{c}}+T_{\mathrm{cool}}}{2}\right)+a_{2} \dot{m}_{\mathrm{air}}+a_{3} \frac{\dot{m}_{\mathrm{air}}}{\dot{m}_{\text {cool }}}
$$

This model based on linear regressors will be called the REG-model.

4.2.3. Validation of temperature models The REGmodel and NTU-model are validated in Figures 5 to 6, with respect to how they predict efficiency. Validation plots for the temperatures are not shown, but NTUmodel gives a maximum error of $8 \mathrm{~K}$ and REG-model $1 \mathrm{~K}$. This shows that REG-model agrees better with measured data compared to NTU-model but it also has more tuning parameters. In both cases, the relative error in temperature is rather small since it should be calculated in absolute temperature (degrees Kelvin). 
Returning to the efficiency as a function of mass flow, we see that the efficiency decreases with increasing $\dot{m}_{\text {air }}$, and that there is knee around $120 \mathrm{~g} / \mathrm{s}$. The NTUmodel predicts a trend that has the converse effect and gives a lower decrease. The result is a systematic error in the output that is $10 \%$ in $\varepsilon$ for high air flows, while the REG-model has an error of less than $3 \%$. A source of error is the fact that the cooling flow $\dot{m}_{\text {cool }}$ is not exactly known since it is estimated from the intercooler fan speed used in the laboratory. However, the erroneous trend with increasing $\dot{m}_{\text {air }}$ would still be present even if the cooling air flow was known. Furthermore, it is interesting to note that larger errors in the efficiency can be tolerated for low flows $\dot{m}_{\text {air }}$ since the temperature increase from $T_{\mathrm{i} c}$ over the compressor is smaller which finally results in smaller errors in $T_{\text {cool }}$. From this it is also understood that it is important to make the least squares fit to minimize the error in $T_{\mathrm{i} c}$ instead of in $\varepsilon$, which also is natural since we want to model the temperature change (note that REG-model is linear in the model parameters in both formulations).
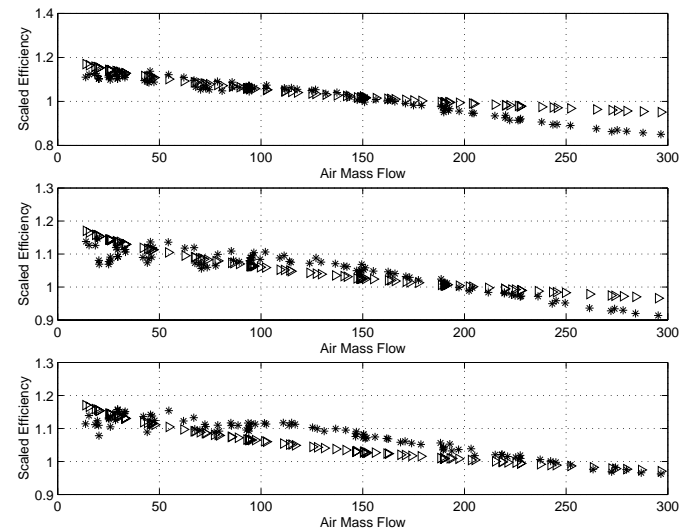

Fig. 5. $\varepsilon$ from NTU-model for three fan speeds. Measured data is represented by $*$ and the model is represented by $\triangle$.
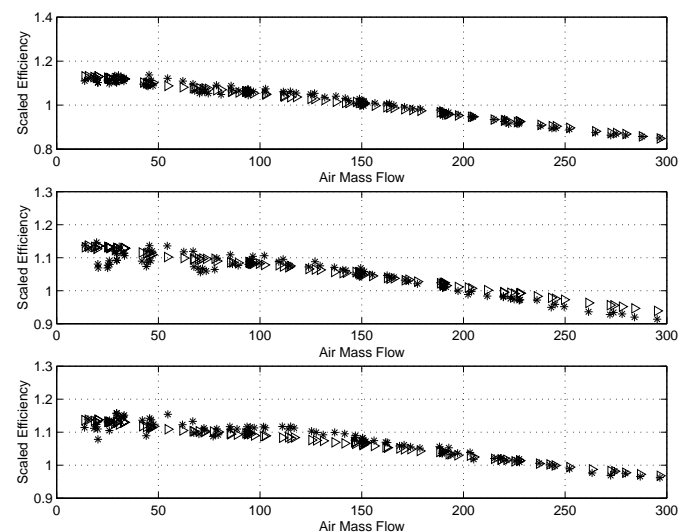

Fig. 6. $\varepsilon$ from REG-model for three fan speeds. Measured data is represented by $*$ and the model is represented by $\triangle$.

\section{THROTTLE AND ENGINE FLOW}

The throttle is modeled using the standard model for isentropic flow through a converging and diverging nossle, see e.g. (Heywood, 1988),

$$
\dot{m}_{a t}\left(\alpha_{t h}, p_{i c}, p_{i m}, T_{i c}\right)=\frac{p_{i c}}{\sqrt{R T_{a}}} Q_{t h}\left(\alpha_{t h}\right) \Psi\left(\Pi_{t h}\right)
$$

where $\Pi_{t h}=\frac{p_{i m}}{p_{i c}}$, and

$$
\begin{aligned}
\Psi\left(\Pi_{t h}^{*}\right) & =\sqrt{\frac{2 \gamma}{\gamma-1}\left(\Pi_{t h}^{*} \frac{2}{\gamma}-\Pi_{t h}^{*} \frac{\gamma+1}{\gamma}\right)} \\
\Pi_{t h}^{*} & =\max \left(\Pi_{t h},\left(\frac{2}{\gamma+1}\right)^{\frac{\gamma}{\gamma-1}}\right)
\end{aligned}
$$

The function $Q\left(\alpha_{t h}\right)$ is a product of the throttle opening area $A$ and the discharge coefficient $C_{D}$, and it is sufficient to model it as a function of the area only, see e.g. (Nyberg and Nielsen., 1997). The function was choosen here as

$$
Q_{t h}=Q_{1}\left(1-\cos \left(a_{2} \alpha^{2}+a_{1} \alpha+a_{0}\right)\right)+Q_{0}
$$

This gives a relative error that is less than $10 \%$, and lower than $5 \%$ for $95 \%$ of the validation data.

The air flow into the cylinders of the engine is modeled using the standard model based on the volumetric efficiency, where $\eta_{\mathrm{vol}}\left(p_{i m}, N\right)$ is stored in a 2dimensional look up table.

$$
\dot{m}_{\mathrm{cyl}}=\frac{\eta_{\mathrm{vol}}\left(p_{i m}, N\right) V_{d} N}{2 R T_{\mathrm{im}}} p_{\mathrm{im}}
$$

\subsection{Exhaust Manifold Out Temperature}

The temperature out of the exhaust manifold before entering the turbine can be modeled with sufficient accuracy as a function of the air mass flow $T_{e m}=$ $f\left(\dot{m}_{\mathrm{af}}\right)$ when operating at stoichiometric conditions.

\section{COMPRESSOR}

Compressor performace is usually presented in a map with scaled variables, where the scaling is based on dimensional analysis. However, for a given turbo machine the geometry does not change, and the influence of the Reynolds number is also considered small, see (Watson and Janota, 1982). Based on this observation, the dimensionless quantities are abandoned for quantites that are referred to a nominal input condition

$$
\begin{aligned}
& \frac{\dot{m} \sqrt{R T_{01}}}{P_{01} D^{2}} \sim \frac{\dot{m} \sqrt{T_{01} / T_{\text {nom }}}}{P_{01} / P_{\text {nom }}}=\dot{m}_{\text {corr }} \\
& \frac{N D}{\sqrt{\gamma R T_{01}}} \sim \frac{N_{\text {eng }}}{\sqrt{T_{01} / T_{\text {nom }}}}=N_{\text {corr }}
\end{aligned}
$$

The new groupings to the left are called corrected mass flow and corrected speed respectively. Compressor performance is determined in a flow bench where 
the compressor is run together with the turbine in a number of points and the state changes of the fluid are measured. From this data a performance map of the compressor is constructed. It is presented in a map with corrected mass flow and pressure ratio on the axis, see Fig. 7.

The shown performance map is normally implemented in some kind of look-up table which serves as the actual compressor model. However, a lookup table is sometimes not suitable. For example, when the model is used to optimize system performance, then it may not be smooth or it may not be invertible. See (Moraal and Kolmanovsky, 1999) for a motivation and longer discussion of some parameterizations that are available. Here, first a standard model for the pressure ratio will be discussed and then models for the air flow.

\subsection{Pressure Model}

If an adiabatic process is assumed, compression from a state with pressure $p_{01}$ and $T_{01}$ to another state, with pressure $p_{02}$ requires an amount of specific energy, $w_{\text {in }}$, that obeys the following relation betwenn the pressure ratio and the specific energy.

$$
\Pi_{c}=\frac{p_{02}}{p_{01}}=\left(1+\frac{w_{\text {in }}}{c_{p} T_{01}}\right)^{\frac{\gamma}{\gamma-1}}
$$

An expression for the work can be derived, see e.g. (Müller et al., 1998), by considering the Euler equations for compressor blades and the losses due to friction and incidence, resulting in

$$
w_{\text {in }}=U_{2}^{2}\left(s_{1}\left(\frac{\dot{m}_{\text {air }}}{U_{2}}\right)^{2}+s_{2}\left(\frac{\dot{m}_{\text {air }}}{U_{2}}\right)+s_{3}\right)
$$

where $U_{2}$ is the rotor tip speed and $s_{i}$ are constants that are tuned to measured data.

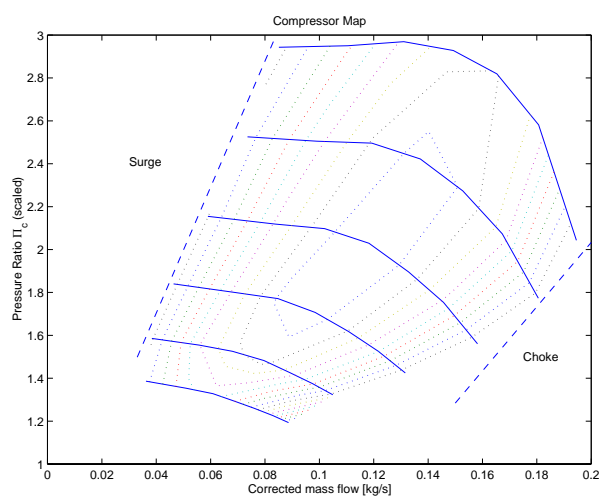

Fig. 7. Typical compressor map. For each speedline (solid) there are two limits to the range of flow. The upper limit is due to choking, when the flow reaches the velocity of sound at some cross section. The lower limit is due to a dangerous instability known as surge. Note that only approximations to the choke and surge limits are given in the plot.

\subsection{Air Mass Flow Model}

In mean value models it is desirable to represent the flow through the compressor as a function of pressure ratio and turbine shaft speed, rather than as in Eqs (8) and (9). To do this, the equations can be inverted to

$$
\dot{m}_{\text {air }}=a_{1} U_{2} \mp \sqrt{a_{2} U_{2}^{2}+a_{3} T_{\text {af }}\left(\Pi_{c}^{\frac{\gamma-1}{\gamma}}-1\right)}
$$

where $a_{i}$ are tuning constants. However, this model is difficult to tune since it produces imaginary numbers for some combinations of parameters and data. Nevertheless, the actual operating range of the compressor is usually limited to a small region of the compressor map, which makes it possible to find a tractable local inverse. Considering this region the following black box model can be used:

$$
\begin{aligned}
\dot{m}_{\text {air }} & =b_{1} \hat{p}_{\mathrm{r}}+b_{2} \hat{p}_{\mathrm{r}}^{2}+b_{3} \hat{p}_{\mathrm{r}}^{3} \\
\hat{p}_{\mathrm{r}} & =\Pi_{c}-b_{4} \hat{N}_{\mathrm{T}}-b_{5} \hat{N}_{\mathrm{T}}^{2} \\
\hat{N}_{\mathrm{T}} & =\frac{N_{\mathrm{T}}-8 \cdot 10^{4}}{2 \cdot 10^{4}}
\end{aligned}
$$

where $b_{i}$ are constants.

\subsection{Efficiency Model}

The efficiency is defined by the ratio of the isentropic and the actual specific input work

$$
\eta_{c}=\frac{w_{i n}}{w_{\text {in }, a c t}}=\frac{\left(\frac{p_{\mathrm{c}}}{p_{\mathrm{af}}}\right)^{\frac{\gamma-1}{\gamma}}-1}{\frac{T_{\mathrm{c}}}{T_{\mathrm{af}}}-1}
$$

where $w_{\text {in }}$ is modeled by Eq. (9). The definition of isentropic efficiency, that is used when the compressure characteristics are measured, is given by the rightmost expression, see (Watson and Janota, 1982). The actual work can be modeled by the following regression model

$$
w_{\text {in }, a c t}=b_{1} \dot{m}_{\text {air }}+b_{2} \dot{m}_{\text {air }} U_{2}+b_{3} U_{2}^{2}+b_{4} U_{2}+b_{5}
$$

Fitting this model to speed lines up to $140000 \mathrm{rpm}$ gives an average error of $0.89 \%$ and a maximum error of $2.25 \%$ in this region. It should be noted that this model is pretty complex and it might be a good idea to use a performance map directly instead.

\subsection{Model Validation}

6.4.1. Temperature increase Eq. (9) gives a model for the specific energy, i.e. the temperature difference (divided by $c_{p}$ ). A validation of this model at stationary conditions is shown in Fig. 8. It can be noted that the average error is low, less than $2 \mathrm{~K}$, but that the model fails for lower temperatures, with errors up to $11 \mathrm{~K}$. A new regression model is introduced here

$$
T_{\text {comp }}=T_{\text {af }}+a_{0}+a_{1} N_{\mathrm{T}}^{2}+a_{2} N_{\mathrm{T}}+a_{3} \dot{m}_{\text {comp }}
$$

Using this model, Eq. (12), considerably reduces the error, as the bottom of the figure shows. For this model 
the average error is less than $1.2 \mathrm{~K}$ and the maximum error less than $6 \mathrm{~K}$.
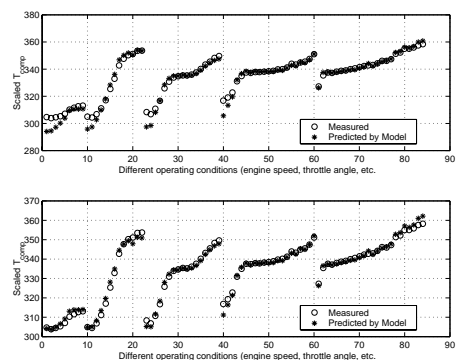

Fig. 8. Validation of temperature model for the compressor at stationary conditions. The upper figure shows validation for model Eq (9) and the lower for Eq (12).

6.4.2. Pressure ratio Figures 9 and 10 show that the model for the pressure ratio is very accurate at stationary conditions (Mean relative error $<0.5 \%$, maximum relative error $\leq 2.6 \%$ ), and it also accurately captures the dynamic behavior, especially at higher engine speeds.
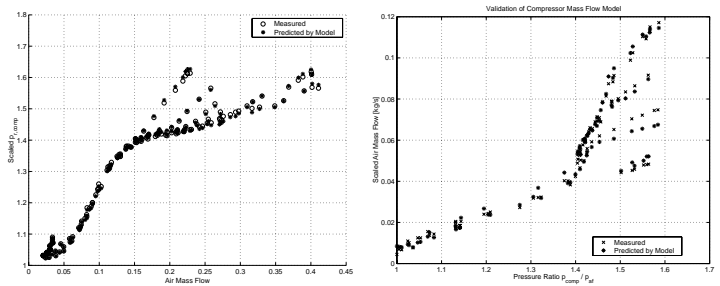

Fig. 9. Validation of pressure model (left) and air flow model (right) for the compressor at stationary engine conditions.

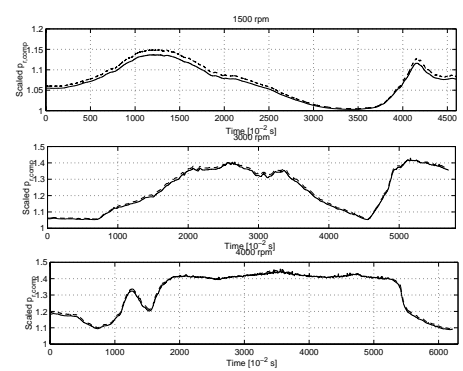

Fig. 10. Validation of pressure model for the compressor at dynamic conditions. The engine speed is held constant, while the throttle angle is varied. Solid - measurement. Dashed - model.

6.4.3. Air mass flow While (10) fails completely due to high sensitivity to input errors, (11) is quite good at stationary conditions, with an average error of $0.0021 \mathrm{~kg} / \mathrm{s}$. This stationary model is quite good for dynamic conditions also, see figure 11 . However, if we compare with the errors for the pressure model, Fig. 9, it is clear that the pressure model gives better aggrement with measured data. The cause for this behavior is that the slope of the speed lines, especially in the lower left corner of Fig. 7, is close to zero which means high sensitivity in air mass flow to changes in pressure ratio. The converse also applies. Furthermore this also motivates that it is desirable to put more efforts into gathering compressor data in the low flow region, especially when developing models for control and simulation.

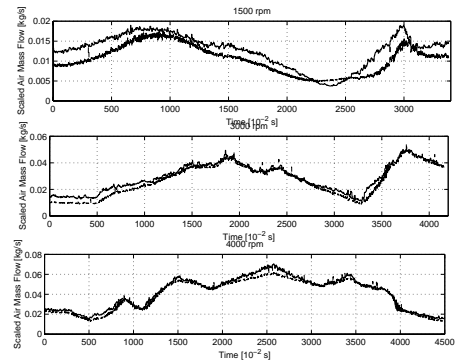

Fig. 11. Validation of air mass flow model for the compressor at dynamic conditions. The engine speed is held constant, while the throttle angle is varied. Solid - measurement. Dashed - model.

\section{TURBINE}

Dimensionless quantities are used when describing the turbine performance, as was done the compressor in Sec. 6. Here it is worth to stress that the dimensionless expressions are of higher importance for the turbine since it is exposed to more extreme variations in inlet conditions compared to the compressor; Temperatures vary from $500 \mathrm{~K}$ up to $1300 \mathrm{~K}$ and pressures at turbine inlet from atmospheric conditions up to $300 \mathrm{kPa}$.

For turbines the corrected mass flow and efficiency is normally plotted versus pressure ratio $\frac{P_{e m}}{P_{t}}=1 / \Pi_{t}$. A typical performance map for a turbine is shown in figure 12. Turbine efficiency is determined from the flow conditions and calculated using

$$
\eta_{t}=\frac{1-\frac{T_{\mathrm{t}}}{T_{\mathrm{em}}}}{1-\left(\frac{p_{\mathrm{t}}}{p_{\mathrm{em}}}\right)^{\frac{\gamma-1}{\gamma}}}
$$

In addition, the mechanical efficiency of the turbocharger might also be included in the turbine efficiency. Look-up tables derived from the map may serve as turbine models but, with the same motivation as for the compressor, it is also interesting to study parameterizations.

\subsection{Pressure Model}

Neglecting the corrected quantities and studying the mass flow alone shows that there exists a relationship between mass flow and pressure ratio for the turbine that is practically independent of turbine shaft speed. Even though the relation is almost linear, the following model is suitable

$$
\Pi_{t}=\frac{p_{t}}{p_{\text {em }}}=1+r_{2} \dot{m}_{t}^{2}+r_{1} \dot{m}_{t}
$$




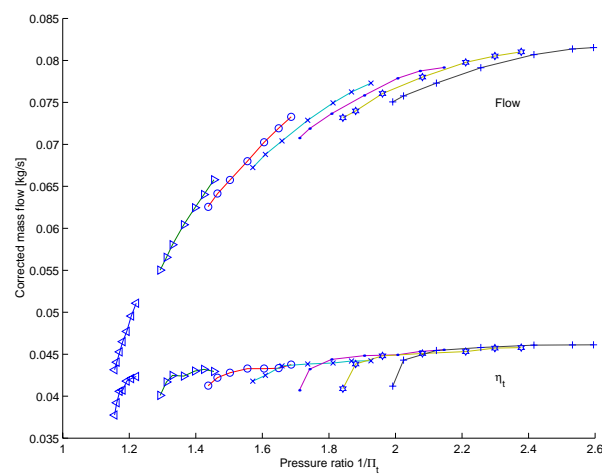

Fig. 12. Typical turbine map, with scaled corrected gas mass flow (upper curves) and efficiency (lower curves), plotted versus the turbine pressure ratio, for several speed lines.

The resulting model (13) has an average error of less than $1.5 \%$, when evaluated on the mapped data. To make the model more general corrected quantities should be used instead of mass flow directly, as explained in earlier sections, however this has not been tested. The parallel flow in the wastegate also has a large effect and makes the validation of the turbine model difficult, when using engine data.

\subsection{Mass Flow Model}

In Fig. 12 it can be seen that the connection between the flow and pressure ratio is almost independent of the compressor speed. Therefore, the speed dependence is neglected and the corrected air mass flow is modeled as a function of only the pressure ratio $\Pi_{t}=\frac{p_{t}}{p_{e m}}$. The following simple model structure produced a better fit to the turbine map compared to the standard model for a restriction Eq. (7) which is normally used also for the turbine flow.

$$
\dot{m}_{t, \text { corr }}=k * \sqrt{1-\Pi_{t}}
$$

This model extrapolates the flow to 0 at $\Pi_{t}=1$.

7.2.1. Validation Fig. 13 shows the model output together with validation data and the turbine map. It can be seen that the model, which has been fitted to the turbine map, gives a lower mass air flow compared to the measured data (look especially in the region $\left.1 / \Pi_{t} \in[1.1,1.25]\right)$. This effect is also seen in (Moraal and Kolmanovsky, 1999, Fig. 10). The effect of opening the waste gate is clearly seen in the region with $1 / \Pi_{t}$ above 1.25 . In the region below 1.25 the waste gate is closed even though this was not possible to check during experiments. Another cause for this effect can be a combination between the temperature sensor location, which is not exactly at the turbine inlet and the high heat transfer in the exhaust. The heat transfer causes the temperature to drop yet some degrees until it reaches the turbine inlet which will change the corrected air mass flow. A third cause is that the static pressure is measured and in the exhaust the density is lower than on the intake side which increases the total pressure and it will influence the corrected air mass flow.

This stresses the point that the turbine map is not sufficient. Additional measurements on the engine have to be made. In this case an increase of $10 \%$ in the model reaches the desired region of the measured engine data. Further measurements in the turbine map at lower flows conditions should also be made since the lowest speed line starts at $1 / \Pi_{t}=1.18$ and the engine data reaches further down.

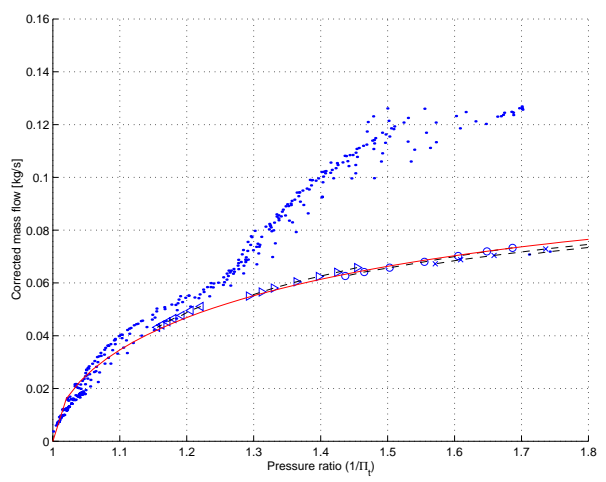

Fig. 13. Validation of the flow model. Dots measurements, solid model, dashed compressor map.

\subsection{Efficiency and Wastegate Models}

Turbine efficiency is important since it determines the power delivered to the compressor. As a first approximation a model that is independent of turbine speed was tested

$$
\eta_{t}=k_{1} \sqrt{\frac{p_{e m}}{p_{t}}-1}+k_{2} \sqrt[4]{\frac{p_{e m}}{p_{t}}-1}+k_{3}
$$

Wastegate flow was modeled using the standard model for a restriction, Eq. (7), with variable area. An antiwindup PI controller was employed, which controls the area such that the intake manifold pressure was limited to a certain value. See (Pettersson, 2000) for a validation of both models.

\subsection{Turbo Shaft Dynamics}

Previous sections described models for compressor and turbine separately and to connect the two submodels Newton's second law for a rotating system is used:

$$
T q_{t}-T q_{c}=I \dot{\omega}
$$

where $T q_{t}$ is the driving torque and $T q_{c}$ denotes braking torque acting on the rotating parts of the turbo charger spinning with the angular velocity $\omega$. The power and torque are connected through $P=T q \omega$ so equation (14) becomes:

$$
P_{t}-P_{c}=I \dot{\omega} \omega
$$


If the turbine and the compressor are treated as two separate thermodynamic systems, $P_{t}$ and $P_{c}$ can be calculated from the first law of thermodynamics. The heat transfer with the surroundings and change in potential energy is neglected.

$$
-P=\dot{m}\left(h_{\text {out }}-h_{\text {in }}\right)=\dot{m} c_{p}\left(T_{\text {out }}-T_{\text {in }}\right)
$$

For the compressor, the net amount of produced power is negative, i.e. the compressor consumes energy. The isentropic relation together with the definition of the compressor isentropic efficiency for $T_{02}-T_{01}$ gives

$$
P_{c}=\dot{m}_{c} c_{p, c} T_{a f} \frac{1}{\eta_{c}}\left(\left(\frac{p_{c}}{p_{a f}}\right)^{\frac{\gamma_{c}-1}{\gamma_{c}}}-1\right)
$$

For the turbine, the net amount of produced energy is positive and the same manipulations yields

$$
P_{t}=\dot{m}_{t} c_{p, t} T_{e m} \eta_{t}\left(\left(\frac{p_{t}}{p_{e m}}\right)^{\frac{\gamma_{t}-1}{\gamma_{t}}}-1\right)
$$

Inserting (18), (17) into (15), yields the final expression for the turbine shaft speed model. Note in the above expressions that the turbo charger friction is included in either $\eta_{t}$ or $\eta_{c}$. It is usually included in $\eta_{t}$, otherwise the power loss by friction must be included explicitly. It has also been reported that by including a friction term some simulation difficulties are avoided.

\section{CONTROL VOLUME DYNAMICS}

Reservoirs are placed in between all flow restricting components and they determine some of the engines dynamic characteristics. Two different models are considered: the standard model with one state variable based on the assumptions of mass conservation and of constant temperature in the reservoir, and a two state model based on energy conservation and mass conservation laws. The standard model is as follows

$$
\frac{d}{d t} p_{\text {res }}=\frac{R T_{\text {res }}}{V_{\text {res }}}\left(\dot{m}_{\text {in }}-\dot{m}_{\text {out }}\right)
$$

and the two state model is as follows

$$
\left\{\begin{array}{lc}
m= & \frac{p V}{R T} \\
\frac{d T}{d t}=\frac{1}{m c_{v}}\left[\dot{m}_{i} c_{v}\left(T_{i}-T\right)+R\left(T_{i} \dot{m}_{i}-T \dot{m}_{o}\right)+\dot{Q}+\dot{W}\right] \\
\frac{d p}{d t}= & \frac{R T}{V} \dot{m}+\frac{m R}{V} \frac{d T}{d t}
\end{array}\right.
$$

\section{CONCLUSIONS}

Turbo charged SI engines are a major possibility in the current trend of down-sized engines to reduce $\mathrm{CO}_{2}$ but still with preserved drivability performance. For control and supervision of these engines it is favorable to have a mean value model, and a component based modeling has therefore been investigated including components as air filter, compressor, after cooler (or intercooler), throttle, engine, turbine, waste gate, and a lumped model for the catalyst and exhaust. The experiments and the model validation have been performed on a Saab 2.31 production engine.
The modeling strategy used throughout the work is to first consider the physics of a component which yields a model structure and then fit parameters in this structure to the model. Often the selected model structures are regression models.

When investigating agreement with measured data and sensitivity of possible model structures, a number of interesting observations were made. Some examples are: The NTU model is not so good. It is important to use dimensionless quantities in turbine models. There is a need for further measurements in the low flow region of turbine maps. A compressor model with pressure ratio as output seems better than an with air flow as output due to sensitivity. However, a tractable local inverse may be found. There is a new regression model for the temperature after the compressor, and so on.

In conclusion, the result of the investigation is a set of model structures that can be fitted to an actual engine (or engine family), and then fulfills the requirements for e.g. observer design.

\section{REFERENCES}

Bergström, Johan and Jan Brugård (1999). Modeling of a turbo charged spark ignited engine. Master's thesis. Linköping University. LiTH-ISYEX-2081.

Guzzella, L., U. Wenger and R. Martin (2000). Ic-engine downsizing and pressure-wave supercharging for fuel economy. SAE Technical Paper 2000-01-1019.

Heywood, J. B. (1988). Internal Combustion Engine Fundamentals. McGraw-Hill series in mechanical engineering. McGraw-Hill.

Holman, J.P. (1992). Heat Transfer, 7th ed, in SI units. McGraw-Hill. ISBN 0-07-112644-9.

Massey, Bernard (1998). Mechanics of Fluids. 7 ed.. Stanley Thornes.

Moraal, Paul and Ilya Kolmanovsky (1999). Turbocharger modeling for automotive control applications. SAE paper 1999-01-0908 pp. 309-322.

Müller, Martin, Elbert Hendricks and Spencer C. Sorenson (1998). Mean Value Modelling of Turbocharged Spark Ignition Engines. SAE SP-1330 Modeling of SI and Diesel Engines (SAE Paper 980784), 125-145.

Nyberg, Mattias and Lars Nielsen. (1997). Model based diagnosis for the air intake system of the SI-engine. SAE Technical Paper (970209).

Pettersson, Fredrik (2000). Simulation of a turbo charged spark ignited engine. Master's thesis. Linköping University, SE-581 83 Linköping.

Watson, N. and M.S. Janota (1982). Turbocharging the Internal Combustion Engine. The Macmillan Press ltd. ISBN 0-333-24290-4. 